\title{
SIEGEL DOMAINS AND REPRESENTATIONS OF JORDAN ALGEBRAS
}

BY

\author{
O. S. ROTHAUS ${ }^{1}$
}

\begin{abstract}
In the analysis of infinitesimal automorphisms of arbitrary Siegel domains, a certain class of nonsemisimple Jordan algebras occurs. The description of all the infinitesimal automorphisms of the domain may be based on a study of representations of the associated Jordan algebra satisfying a certain "strange identity". In this paper, all the possibilities for the Jordan algebra and representations satisfying the identity are given.
\end{abstract}

0. The infinitesimal automorphisms of arbitrary Siegel domains are now rather well understood as a result of the investigations in [2, 4-9], save for some technical questions concerning representations of Jordan algebras satisfying a "strange" identity $[\mathbf{8}, 9]$. In this paper we will elucidate these remaining questions, drawing freely on the definitions, notations, and results of our papers [6-8].

$U$ is a real finite dimensional vector space, $U_{\mathbf{C}}=U \otimes \mathbf{C}$ its complexification. $\Omega$ is a regular convex cone in $U . V$ is a finite dimensional complex vector space, and $F(\cdot, \cdot)$, complex linear in the first variable, is an $\Omega$-Hermitian form on $V$. The set of points $D=\left\{(u, v) \in U_{\mathrm{C}} \times V \mid \operatorname{Im} u-F(v, v) \in \Omega\right\}$ is a Siegel domain of Type II.

Let $g$ denote the Lie algebra of the group $G$ of holomorphic automorphisms of $D$. As is well known $[4,5,8], g$ is graded.

$$
g=g_{-1} \oplus g_{-1 / 2} \oplus g_{0} \oplus g_{1 / 2} \oplus g_{1} \text {, and any } X \in g_{1} \text { may be written }
$$

$$
X=R(u) u \frac{\partial}{\partial u}+S(u) v \frac{\partial}{\partial v}
$$

(there is a slight notational change from that in [8]). For an $X$ of the above form, the necessary and sufficient conditions that $X \in g_{1}$ are that:

(1) The multiplication $u \circ u^{\prime}=R(u) u^{\prime}$ endows $U$ with structure $\mathscr{Q}$ of real Jordan algebra,

(2) The map $u \rightarrow S(u)$ is a representation of $\mathscr{Q}$ in End $V$; i.e.

$$
S\left(u \circ u^{\prime}\right)=\frac{1}{2} S(u) S\left(u^{\prime}\right)+\frac{1}{2} S\left(u^{\prime}\right) S(u) .
$$

(When necessary, we extend the representation to the complexification of $Q$.)

(3) $(\Theta, \Omega)$ is a Jordan pair, and $S$ is $F$-related to $Q$, i.e.

$$
a \circ F(v, w)=\frac{1}{2} F(S(a) v, w)+\frac{1}{2} F(v, S(a) w),
$$

Received by the editors November 30, 1979 and, in revised form, December 9, 1980.

1980 Mathematics Subject Classification. Primary 17C15, 32A07; Secondary 17B60, 32M05.

'This research was supported in part by NSF grants MCS-7810470 and MCS-7718723. 
and

(4) $F(S(F(v, w) v, w))=F(v, S(F(w, v) w))$.

A 5-tuple ( $Q, \Omega, V, S, F)$ satisfying (1) to (4) above will be called an admissible data set. (The notion of Jordan pair in (3) above should not be confused with that introduced by O. Loos in Jordan pairs, Lecture Notes in Math., vol. 460, SpringerVerlag).

Number (4) above is the so-called "strange" identity, whose implications we explore in this paper. The reader will soon see that it carries a considerable amount of structural information. (A study of the case in which $Q$ is semisimple is carried out in $[1,9]$.)

The general structure of the algebra $Q$ is revealed in [6-8]. It is known to contain a principal idempotent $e$, with respect to which it has a Pierce decomposition $Q=Q$, $\oplus \Theta_{1 / 2} \oplus \mathbb{Q}_{0}$ such that:

(1) $\Theta_{1}$ is semisimple and $e$ is the unit,

(2) $\operatorname{rad} Q=\mathbb{Q}_{1 / 2} \oplus \mathbb{Q}_{0}$,

(3) $\mathbb{Q}_{1 / 2}$ is stable under multiplication by $\mathscr{Q}_{1}$, and

(4) Multiplication by $\mathbb{Q}_{0}$ always gives 0 .

We define $P(r, s)=r \circ s, r, s \in \mathbb{Q}_{1 / 2}$. Define $H(x)$ for $x \in \mathbb{Q}_{1}$ as the endomorphism of $\mathbb{U}_{1 / 2}$ given by $H(x) r=2 x \circ r$. Then $x \rightarrow H(x)$ is a real unital representation of $\Theta_{1}$, and $P(H(x) r, s)=P(r, H(x) s)$.

We let $\Omega_{1}$ and $\Omega_{0}$ be the projections of $\Omega$ into $\Theta_{1}$ and $\Theta_{0}$ respectively. Then $\Omega_{1}$ and $\Omega_{0}$ are regular convex cones in $\mathbb{Q}_{1}$ and $\mathcal{Q}_{0}$. Moreover, $\Omega$ is precisely the set of points $x+r+k, x \in \Omega_{1}, r \in \mathbb{Q}_{1 / 2}, k \in \mathbb{Q}_{0}$, such that $k-P\left(H^{-1}(x) r, r\right) \in \Omega_{0}$.

It will be useful for us later to note now that the Jordan pair $\left(\mathbb{Q}_{0}, \Omega_{0}\right)$ - a pair in our sense even though $\mathbb{Q}_{0}$ is a trivial algebra - is uniquely determined by $\left(Q_{,}, \Omega\right)$. For if $e^{\prime}$ is another principal idempotent for $Q$, it is known [6] that there exists $r \in Q_{1 / 2}$ such that $e^{\prime}=(\exp R(r)) e$, and $\exp R(r)$ is both an automorphism of $\leftrightarrow$ and an automorphism of $\Omega$.

We will call $\left(\Theta_{0}, \Omega_{0}\right)$ the base of $(Q, \Omega)$.

It is known $[7,8]$ that the element $X \in g_{1}$ may be selected as a "maximal" element, which guarantees that $X$ together with $g_{-1} \oplus g_{-1 / 2} \oplus g_{0}$ generates all of $g$. And also $X$ may be chosen to insure that $Q_{1}$ is a compact Jordan algebra, and $\Omega_{1}$ is the associated domain of positivity; i.e. $\left(Q_{1}, \Omega_{1}\right)$ is a positive pair [7]. We will assume throughout this paper that $X$ has been selected to satisfy the latter requirement. In this event, it is easy to see that $\bar{\Omega}$ (closure of $\Omega$ ) is precisely the set of points $(x+r)^{2}+k$, where $x \in \mathbb{Q}_{1}, r \in \mathbb{Q}_{1 / 2}$, and $k \in \bar{\Omega}_{0}$.

Our strategy in this paper is essentially as follows. Given the algebra $Q$, principal idempotent $e$, and the representation $S$ in End $V$, since $S(e)$ is idempotent, we have an eigenspace decomposition $V=V_{1} \oplus V_{0}$. Using additionally the existence of the $\Omega$-Hermitian form $F$, it follows that, [8], $S\left(\Theta_{1}\right) V_{1} \subset V_{1}, S(\Theta) V_{0}=0, S\left(\Theta_{1 / 2}\right) V_{1} \subset V_{0}$, and $S\left(Q_{0}\right)=0$. A representation of $Q$ satisfying the four above requirements will be called special. It is clear that $F\left(V_{1}, V_{1}\right) \in \mathbb{Q}_{1} \otimes C, F\left(V_{1}, V_{0}\right) \in \mathbb{Q}_{1 / 2} \otimes C$, and $F\left(V_{0}, V_{0}\right) \in \mathbb{Q}_{0} \otimes C$.

Because $Q$ is semisimple, the possibilities for the form $F$ restricted to $V_{1}$ can be described precisely. We investigate the remaining necessary and sufficient conditions 
on $Q$ and the special representation $S$ which insure that a given restriction of $F$ on $V_{1}$ can be extended to an admissible one on all of $V$.

To accommodate this strategy, we introduce the following notation. Let $(\mathscr{Q}, \Omega)$ be a positive Jordan pair, and $S$ a special representation of $\mathscr{Q}$ in complex vector space $V$. Given a principal idempotent $e$, we split $V=V_{1} \oplus V_{0}$. $\left(V_{0}\right.$ does not depend, as a matter of fact, on the choice of e.) We suppose, moreover, we are given a $\Omega_{1}$-Hermitian form $F$ on $V_{1}$ such that $S$ is $F$-related to $\mathbb{Q}_{1}$ and satisfies the strange identity. We will say then we have an incomplete data set $(Q, \Omega, V, S, F)$ and will call the incomplete data set admissible if the definition of $F$ can be extended to all of $V$, and with the extended $F$ the data set becomes admissible.

With the extended $F$ in hand, we have a Siegel domain $D$, and an element $X \in g_{1}$ which gives us back both $Q$ and $S$. We postpone to a subsequent paper the question whether the $X$ we thus arrive at is indeed maximal for the given domain $D$.

1. In this section we are going to limit the possibilities for $V_{0}$, and reduce as well to the case $\mathcal{Q}_{1}$ simple.

Define $V_{0}^{\prime}=S\left(\mathscr{Q}_{1 / 2}\right) V_{1}$. We know $V_{0}^{\prime} \subset V_{0}$. Let $\rho$ be any linear form in the interior of the dual cone to $\Omega_{0}$, and consider the Hermitian definite form $Q(\cdot, \cdot)$ on $V_{0}$ given by $Q(\cdot, \cdot)=\rho(F(\cdot, \cdot))$. Let $V_{0}^{\prime \prime}$ be the orthocomplement of $V_{0}^{\prime}$ with respect to $Q$. Clearly $V_{0}=V_{0}^{\prime} \oplus V_{0}^{\prime \prime}$.

Lemma. $V_{0}^{\prime \prime}=\left\{c \in V_{0} \mid F(v, c)=0 \forall v \in V_{1}\right\}$.

For the proof, define $W=\left\{c \in V_{0} \mid F(v, c)=0 \quad \forall v \in V_{1}\right\}$. If $c \in V_{0}^{\prime \prime}$, then $Q(S(r) v, c)=\rho(F(S(r) v, c))=0$ for $r \in \mathbb{Q}_{1 / 2}, \quad v \in V_{1}$. But $F(S(r) v, c)=$ $2 r \circ F(v, c)$. It is known [7] that for any nonzero $r \in \mathbb{Q}_{1 / 2}$ that $r^{2} \in \bar{\Omega}_{0}-0$, so $\rho\left(r^{2}\right) \neq 0$. Putting in turn then $r$ equal to the real and imaginary parts of $F(v, c)$, we conclude $F(v, c)=0$. Hence $c \in W$.

If $c \in W$, then $F(v, c)=0$ for all $v \in V_{1} \Rightarrow r \circ F(v, c)=0$ for all $r \in \mathbb{Q}_{1 / 2}$, $\Rightarrow F\left(V_{0}^{\prime}, c\right)=0, \Rightarrow c \in V_{0}^{\prime \prime}$, completing the proof.

Now put $V^{\prime}=V_{1} \oplus V_{0}^{\prime}$, so that $V=V^{\prime} \oplus V_{0}^{\prime \prime}$. If $v=v^{\prime}+v_{0}^{\prime \prime}, w=w^{\prime}+w_{0}^{\prime \prime}$, then $F(v, w)=F\left(v^{\prime}, w^{\prime}\right)+F\left(v_{0}^{\prime \prime}, w_{0}^{\prime \prime}\right)$. Thus $F$ is $\Omega$-Hermitian if and only if its restrictions to $V^{\prime}$ and $V_{0}^{\prime \prime}$ are, and, as is readily verified, will satisfy the strange identity if and only if its restriction to $V^{\prime}$ does.

It is now clear that the role played by $V_{0}^{\prime \prime}$ is superfluous. For the sequel then, we will always assume $V_{0}^{\prime \prime}=0$; i.e. we may identify $V_{0}$ with $S\left(\mathbb{Q}_{1 / 2}\right) V_{1}$. Put another way, we will assume $S(\mathbb{Q}) V=V$, which shows the requirement independent of the choice of principal idempotent. Accordingly, we will call $(Q, \Omega, V, S, F)$ a reduced admissible data set if $S(\mathbb{Q}) V=V$. All data sets hereafter occurring will be assumed reduced. The notion of reduced data set also being applicable to incomplete sets, we will also assume all incomplete data sets are reduced as well.

Next, we want to reduce to the case $\mathscr{Q}_{1}$ simple. Write $\mathbb{Q}_{1}=\bigoplus_{1}^{p} \mathbb{Q}_{1}(i)$, the decomposition of $\mathbb{Q}_{1}$ into simple compact components. Put $e=\sum_{p} e(i)$, the corresponding decomposition of the unit element of $\mathbb{Q}_{1}$. Since $H(e(i))$ are a family of orthogonal idempotents summing to the identity operator, we get a joint eigenspace decomposition $\mathbb{Q}_{1 / 2}=\bigoplus_{1}^{p} \mathbb{Q}_{1 / 2}(i)$. It follows readily that $\mathbb{Q}_{1 / 2}(i) \circ \mathbb{Q}_{1 / 2}(j)=0$ and $\mathbb{Q}_{1}(i) \circ \mathbb{Q}_{1 / 2}(j)=0$ if $i \neq j$. Put $\mathbb{Q}(i)=\mathbb{Q}_{1}(i) \oplus \mathbb{Q}_{1 / 2}(i) \oplus \mathbb{Q}_{0}$, and $\Omega(i)$ the 
relative interior of the set $a^{2}+k, a \in \mathbb{Q}(i), k \in \bar{\Omega}_{0}$. Then it is easy to see that $(\mathbb{Q}(i), \Omega(i))$ is a Jordan pair with base $\left(\mathbb{Q}_{0}, \Omega_{0}\right)$.

Since $S(e(i))$ are a family of orthogonal idempotents summing to the identity on $V_{1}$, we obtain a joint eigenspace decomposition $V_{1}=\oplus_{1}^{p} V_{1}(i)$. It is clear that $F\left(V_{1}(i), V_{1}(j)\right)=0$ if $i \neq j$; moreover, $F\left(V_{1}(i), V_{1}(i)\right) \in \mathbb{Q}_{1}(i) \otimes \mathbf{C}$. Also, if $r \in$ $Q_{1 / 2}(i), v \in V_{1}(j)$, then $S(r) v=0$ if $i \neq j$. For $S(r) v=2 S(e(i) \circ r) v=$ $S(r) S(e(i)) v+S(e(i)) S(r) v=0+0=0$. So put $V_{0}(i)=S\left(\Theta_{1 / 2}(i)\right) V_{1}(i)$, and put $V(i)=V_{1}(i) \oplus V_{0}(i)$. Clearly now, $V_{0}=\oplus_{1}^{p} V_{0}(i)$, and also $F(V(i), V(j))=0$ if $i \neq j$, while $F(V(i), V(i)) \in \mathbb{Q}(i) \otimes \mathbf{C}$.

Let $S(i)$ be the restriction of $S$ to $V(i)$ and $F(i)$ the restriction of $F$ to $V(i)$. Our remarks above imply that $(\mathbb{Q}(i), \Omega(i), V(i), S(i), F(i))$ is a reduced admissible data set, and the base of $(\mathscr{Q}(i), \Omega(i))$ is $\left(\mathbb{Q}_{0}, \Omega_{0}\right)$. If we have, on the other hand, a collection of reduced admissible data sets all over a common base $\left(\mathbb{Q}_{0}, \Omega_{0}\right)$, they can obviously be reassembled by reversing the analysis above. Consequently we confine our attention in the sequel to reduced admissible data sets, or reduced incomplete data sets, for which $\mathbb{Q}_{1}$ is simple compact.

If $(\leftrightarrow, \Omega, V, S, F)$ is reduced admissible or reduced incomplete, and $V_{1}=\{0\}$, then $S\left(\Theta_{1 / 2}\right) V_{1}=V_{0}=\{0\}$, so $V=\{0\}$ and $F \equiv 0$. To avoid this trivial case, we suppose in the sequel all data sets have $V \neq\{0\}$.

In summary then, all our data sets, unless otherwise noted, whether complete or incomplete, will be assumed reduced, to have $V \neq\{0\}$, to have $\mathbb{Q}_{1}$ simple and $\left(\Theta_{1}, \Omega_{1}\right)$ a positive pair.

2. We will need shortly a description of all admissible data sets $(Q, \Omega, V, S, F)$ for which $\Theta$ is simple compact, $\Omega$ is the associated domain of positivity. It will also be convenient to list, for each simple $\mathscr{Q}$ appearing in an admissible data set, its distinct nontrivial irreducible real representations, denoted $h$, operating in real vector space $U$, and its distinct nontrivial irreducible complex representations, denoted $s$, operating in complex vector space $W$. Each irreducible complex representation has a unique (up to real scalar multiple) invariant Hermitian form, denoted $f$.

$F(\cdot, \cdot)$ being an element of $\Theta \otimes_{\mathbf{R}} \mathbf{C}$ we write where convenient, $F(v, w)=F_{1}(v, w)$ $\otimes 1+F_{2}(v, w) \otimes i$.

The relevant facts can be extracted from $[1,9]$, but see also $[2,3]$.

(I) $Q=\mathbf{R}, e$ the unit, $V=\mathbf{C}^{p}, S(x) v=x v, F(v, w)=Q(v, w) e, Q$ any positive Hermitian form on $\mathbf{C}^{p}$,

$$
\begin{array}{rlrl}
U & =\mathbf{R}, & h(x) u=x u, \\
W & =\mathbf{C}, \quad s(x) w=x w, \quad f(v, w)=v \bar{w} .
\end{array}
$$

(II) $\mathbb{Q}=n \times n$ complex Hermitian matrices, $n \geqslant 2$. (We identify $\mathbb{Q} \otimes_{\mathbf{R}} \mathbf{C}$ with $n \times n$ complex matrices, the conjugation being transpose conjugate.) $V=\mathbf{C}^{n} \otimes_{\mathbf{C}} \mathbf{C}^{p}$,

(i) $S(x)(v \otimes \alpha)=x v \otimes \alpha, F(v \otimes \alpha, w \otimes \beta)=Q(\alpha, \beta) v w^{*}$, or

(ii) $S(x)(v \otimes \alpha)=\bar{x} v \otimes \alpha, F(v \otimes \alpha, w \otimes \beta)=Q(\alpha, \beta) \bar{w} v^{t}$. In both cases $Q$ is any positive Hermitian form on $\mathbf{C}^{p}$.

$$
\begin{array}{ll}
U=\mathbf{R}^{n}, & h(x) u=x u, \\
W=\mathbf{C}^{n}, & \text { (i) } s(x) w=x w, \quad f(v, w)=w^{*} v, \quad \text { or } \\
& \text { (ii) } s(x) w=\bar{x} w, \quad f(v, w)=w^{*} v .
\end{array}
$$


(III) $\mathbb{Q}=n \times n$ quaternionic Hermitian matrices, $n \geqslant 3 . V=\mathbf{H}^{n}$ (complex scalars acting on right), $S(x) v=x v . F_{1}(v, w)=\eta\left(v w^{*}+w v^{*}\right)$, and $F_{2}(v, w)=F_{1}(v, w i), \eta$ any positive scalar

$$
\begin{aligned}
U & =\mathbf{H}^{n}, & & h(x) u=x u, \\
W & =\mathbf{H}^{n}, & & s(x) w=x w, f(v, w)=w^{*} v-i w^{*} v i .
\end{aligned}
$$

(IV) $\mathbb{Q}=2 \times 2$ quaternionic Hermitian matrices, $V, S$, and $F$ as in (III) above. But also,

$$
\begin{array}{ll}
U=\mathbf{H}^{2}, & \text { (i) } h(x) u=x u \text { or } \\
& \text { (ii) } h(x) u=\bar{x} u, \\
W=\mathbf{H}^{2}, & \text { (i) } s(x) w=x w, \quad f(v, w)=w^{*} v-i w^{*} v i, \quad \text { or } \\
& \text { (ii) } s(x) w=\bar{x} w, f(v, w)=w^{*} v-i w^{*} v i .
\end{array}
$$

(V) $\mathbb{Q}=$ Jordan algebra of dimension 8 based on a sum of seven squares. Since we do not need details here, we give only general description. There is only one nontrivial real irreducible representation, which acts in $\mathbf{C}^{8}$. This real representation splits over the complexes into two inequivalent complex representations, each acting in $\mathbf{C}^{8}$, each of which leads to reduced admissible data sets with a uniquely determined (up to real scalar multiple) $\Omega$-Hermitian form. There are no other reduced admissible data sets.

3. Let $(Q, \Omega, V, S, F)$ be a five tuple which satisfies all the requirements for admissibility except the strange identity. We construct, as usual, the subspaces $V_{1}$ and $V_{0}$. Then:

LEMma. The necessary and sufficient conditions that $F$ satisfy the strange identity $F(S(F(v, c)) v, c)=F(v, S(F(c, v)) c)$ are

(1) it is true when both $v$ and $c \in V_{1}$,

(2) if $v \in V, c \in V_{0}$, then $S(F(v, c)) v=0$.

If the strange identity holds, then obviously (1) holds. But the strange identity is known [8] to be equivalent to the somewhat stronger statement $F(S(F(v, c)) v, d)=$ $F(v, S(F(d, v)) c)$. If we pick $c$ in $V_{0}$, the right-hand side is zero since $V_{0}$ is annihilated by $S$. But then the vanishing of the left-hand side for any $d$ implies $S(F(v, c)) v \equiv 0$.

Now supposing (1) and (2) are satisfied, we must show $F(S(F(v, c)) v, c)=$ $F(v, S(F(c, v)) v)$. Writing $c=c_{1}+c_{0}, c_{i} \in V_{i}$, it will suffice to show that $F\left(S\left(F\left(v, c_{1}\right)\right) v, c_{0}\right)=F\left(v, S\left(F\left(c_{0}, v\right)\right) c_{1}\right)$, and $F\left(S\left(F\left(v, c_{1}\right)\right) v, c_{1}\right)=$ $F\left(v ; S\left(F\left(c_{1}, v\right)\right) c_{1}\right)$. The first equality above follows by computing $F\left(v, c_{1}\right) \circ F\left(v, c_{0}\right)$ in two different ways, using commutativity of Jordan multiplication. Writing $v=v_{1}$ $+v_{0}$, the second will hold if we show $F\left(S\left(F\left(v_{0}, c_{1}\right)\right) v_{1}, c_{1}\right)=F\left(v_{0}, S\left(F\left(c_{1}, v_{1}\right)\right) c_{1}\right)$, which follows as before by computing $F\left(v_{1}, c_{1}\right) \circ F\left(v_{0}, c_{1}\right)$ in two different ways.

COROLlary. The second condition in the last lemma may be replaced by $\left(2^{\prime}\right)$ $F(v, c) \circ F(v, c)=0$ if $v \in V, c \in V_{0}$.

For if (2) holds then $F(v, c) \circ F(v, c)=\frac{1}{2} F(S(F(v, c)) v, c)=0$. While if $\left(2^{\prime}\right)$ holds, then by polarizing, $F(v, c) \circ F(v, d)=0$ if $v \in V_{1}, c, d \in V_{0}$. And then 
$0=F(v, c) \circ F(v, d)=\frac{1}{2} F(S(F(v, c)) v, d)$. Since $S(F(v, c)) v \in V_{0}$, we conclude $S(F(v, c)) v=0$.

As a consequence of this lemma we see that with $S$ restricted to $\mathscr{Q}_{1}$, and $F$ restricted to $V_{1},\left(Q_{1}, \Omega_{1}, V_{1}, S, F\right)$ is admissible. Consequently, we need only consider $Q_{1}$ in one of the cases described in $\S 2$. The remainder of the paper studies these five cases. Before proceeding, however, we need a few more results.

The next pair of lemmas cover a technical situation we meet repeatedly. Let $\mathbb{Q}=\mathbb{Q}_{1} \oplus \mathbb{Q}_{1 / 2} \oplus \mathbb{Q}_{0}$ be one of the algebras we have been considering, and let $S$ be a special representation of $\mathscr{Q}$ in a vector space $V, V=V_{1} \oplus V_{0}$ as usual. Let $s$ and $s^{\prime}$ be two complex irreducible representations of $Q_{1}$ in $W$ and $W^{\prime}$ respectively, the first having invariant Hermitian form $f(\cdot, \cdot)$. Suppose, moreover, that $s$ and $s^{\prime}$ are real irreducible when $W$ and $W^{\prime}$ are viewed as real vector spaces. Furthermore, we suppose that $S$, restricted to $V_{1}$, contains $s$ as a subrepresentation. And $\mathscr{Q}_{1 / 2}$, which is a real representation space for $\mathbb{Q}_{1}$, contains $s^{\prime}$ operating in $W^{\prime}$ as a real subrepresentation.

Lemma. Let $r \in W^{\prime}$ be an element of $\Theta_{1 / 2}$ and $w \in W$ be an element of $V_{1}$. If $s$ and $s^{\prime}$ are inequivalent as real representations then $S(r) w=0$.

Let $\theta$ be any linear form in the dual space to $V_{0}$ considered as a real vector space, and consider the real bilinear form $Q(\cdot, \cdot)$ on $W^{\prime} \times W$ defined by $Q(r, w)=$ $\theta(S(r) w)$. If $f_{1}$ is the real part of $f$, there exists a real linear map $L: W^{\prime} \rightarrow W$ such that $Q(r, w)=f_{1}(L r, w)$. But for $x \in \mathbb{Q}_{1}$,

$$
\begin{aligned}
S\left(s^{\prime}(x) r\right) w & =2 S(x \circ r) w=S(x) S(r) w+S(r) S(x) w \\
& =S(r) S(x) w=S(r) s(x) w,
\end{aligned}
$$

whence $Q\left(s^{\prime}(x) r, w\right)=Q(r, s(x) w)$, from which it follows that $L s^{\prime}=s L$. By Schur's lemma, $L=0$, which gives the conclusion of the lemma.

Continuing the notation of the last lemma, we next suppose $W=W^{\prime}, s=s^{\prime} . W$, considered as real representation space for $s$, always has $s$-equivariant complex structure. It may have $s$-equivariant quaternionic structure. Then:

Lemma. If $W$ has only s-equivariant complex structure there exists unique $\lambda \in V_{0}$ such that $S(r) w=f(w, r) \lambda$. If $W$ has s-equivariant quaternionic structure, there exist unique $\lambda$ and $\rho \in V_{0}$ such that $S(r) w=f(w, r) \lambda+f(w, r j) \rho$.

Suppose $W$ has only s-equivariant complex structure. Consider real valued bilinear forms on $W \times W$ ( $W$ considered as real vector space) which are $s$ invariant. It is easy to see that a real basis for such forms is given by $f_{1}\left(w, w^{\prime}\right)$ and $f_{1}\left(w, i w^{\prime}\right)$.

Repeating the analysis of the last lemma, we infer the unique existence of $\rho_{1}$ and $\rho_{2} \in V_{0}$ such that $S(r) w=f_{1}(w, r) \rho_{1}+f_{1}(w, i r) \rho_{2}$. But since $S(r) w$ is $\mathbf{C}$ linear in the variable $w, \rho_{2}=i \rho_{1}$, and the desired conclusion follows.

If $W$ has $s$-equivariant quaternionic structure, then the real bilinear forms on $W \times W$ which are $s$ invariant have as basis $f_{1}\left(w, w^{\prime}\right), f_{1}\left(w, w^{\prime} i\right), f_{1}\left(w, w^{\prime} j\right)$, and $f_{1}\left(w, w^{\prime} k\right)$. The analysis now proceeds as in the former case. 
The last lemma of this section refers to an arbitrary admissible data set $(Q, \Omega, V, S, F)$, not necessarily satisfying any of the special stipulations we have introduced.

Lemma. Let $v \in V, r \in \mathbb{Q}_{1 / 2}$. Then $S(r) v=0$ if and only if $r \circ F(v, v)=0$.

For $r \circ F(v, v)=\frac{1}{2} F(S(r) v, v)+\frac{1}{2} F(v, S(r) v)$, so $S(r) v=0 \Rightarrow r \circ F(v, v)=0$. On the other hand, $r \circ(r \circ F(v, v))=\frac{1}{2} F(S(r) v, S(r) v)$; since $F(w, w)=0$ if and only if $w=0$, the reverse implication is clear.

4. Let $(\mathbb{Q}, \Omega, V, S, F)$ be an admissible data set with $\mathscr{Q}_{1}$ the Jordan algebra in case $\mathrm{V}$ of $\S 2 . V_{1}$ may be taken as the action space of either of the two complex irreducible representations of $\mathbb{Q}_{1}$, say $s$ acting in $W \approx \mathbf{C}^{8} . S$ restricted to $\mathbb{Q}_{1}$ is $s$. For $\mathbb{Q}_{1 / 2}$ we may take $\mathbb{Q}_{1 / 2}=W \otimes_{\mathbf{R}} \mathbf{R}^{p}$; if $x \in \mathbb{Q}_{1}$ and $r=w \otimes \alpha \in \mathbb{Q}_{1 / 2}$, then $x \circ r=\frac{1}{2} s(x) w$ $\otimes \alpha$. From a lemma of the last section, we know for $v \in V_{1}$ that $S(w \otimes \alpha) v=$ $\lambda(\alpha) f(v, w)$, where $\lambda(\alpha) \in V_{0}$.

Select $e_{1}$ and $e_{2}$ primitive orthogonal idempotents in $Q_{1}, e=e_{1}+e_{2}$. Select $v \neq 0$ in $V_{1}$ such that $S\left(e_{1}\right) v=s\left(e_{1}\right) v=v$. It is clear that for this $v, F(v, v)=\eta e_{1}$, for some $\eta>0$. Select if possible $\alpha$ so that $\lambda(\alpha) \neq 0$. Then for $w \otimes \alpha \in \mathbb{Q}_{1 / 2}$, $S(w \otimes \alpha) v=0$ if and only if $f(v, w)=0$, and by the last lemma of the last section, if and only if $(w \otimes \alpha) \circ F(v, v)=0$; i.e., if and only if $s\left(e_{1}\right) w=0$. Since $e_{1}$ and $e_{2}$ may be interchanged by an automorphism of $Q_{1}$, and there is only one real irreducible representation of $Q_{1}$, it is clear that the null space of $s\left(e_{1}\right)$ is of complex dimension 4. But the complex dimension of the set of $w \in W$ such that $f(v, w)=0$ is 7 .

This contradiction shows that $\lambda(\alpha) \equiv 0$, and by our convention on $V_{0}$ that $V_{0}=\{0\}$. Thus $S$ restricted to $\mathbb{Q}_{1 / 2}$ is trivial. We will show $\mathbb{Q}_{1 / 2}$ must be zero. Let $F(v, w)=F_{1}(v, w) \otimes 1+F_{2}(v, w) \otimes i$, the decomposition into real and imaginary parts. We know for all $r \in \mathbb{Q}_{1 / 2}$ that $r \circ F_{1}(v, w)=0$. But the real linear span of $F_{1}(v, w)$ is an ideal in $\mathscr{Q}_{1}$; since $\mathscr{Q}_{1}$ is simple, $e$ belongs to this ideal. Hence $r \circ e=\frac{1}{2} r=0$.

Thus $\mathbb{Q}_{1 / 2}=\{0\}$. We now have

THEOREM. Let $(\&, \Omega, V, S, F)$ be an incomplete data set with $\mathscr{Q}_{1}$ the Jordan algebra of case $\mathrm{V}$. Then the data set may be completed if and only if $Q_{1 / 2}=\{0\}$.

We have proved the necessity above. The sufficiency is obvious.

5. Now we take an admissible data set $(\mathscr{Q}, \Omega, V, S, F)$ with $\mathcal{Q}_{1}$ in case II. For $V_{1}$ we may take $\mathbf{C}^{n} \otimes_{\mathbf{C}} \mathbf{C}^{p}$ and for $x \in \mathbb{Q}_{1}, v \otimes \beta \in V_{1}$ we take either the action (i) $S(x)(v \otimes \beta)=x v \otimes \beta$ or (ii) $S(x)(v \otimes \beta)=\bar{x} v \otimes \beta$. Since the discussion in the two cases is parallel we stick to the first. For $Q_{1 / 2}$ we may take $\mathbf{C}^{n} \otimes_{\mathbf{R}} \mathbf{R}^{q}$, and for $x \in \mathbb{Q}_{1}, r \otimes \alpha \in \mathbb{Q}_{1 / 2}, x \circ(r \otimes \alpha)=\frac{1}{2} x r \otimes \alpha$. The complexification of $\mathbb{Q}_{1 / 2}$ will be identified with $\mathbf{C}^{n} \otimes_{\mathbf{R}} \mathbf{R}^{q} \otimes_{\mathbf{R}} \mathbf{C}$, the last factor in the tensor product carrying both the complex structure and the conjugation. 
By a lemma of $\S 3$, we know $S(r \otimes \alpha)(v \otimes \beta)=\left(r^{*} v\right) \lambda(\alpha, \beta)$ for a unique $\lambda(\alpha, \beta) \in V_{0}$. Hence $S($ ir $\otimes \alpha)(i v \otimes \beta)=S(r \otimes \alpha)(v \otimes \beta)$. It follows that

$$
S(r \otimes \alpha \otimes 1+i r \otimes \alpha \otimes i)(v \otimes \beta)=2 S(r \otimes \alpha)(v \otimes \beta),
$$

while $S(r \otimes \alpha \otimes 1-i r \otimes \alpha \otimes i)(v \otimes \beta)=0$.

Let $w \otimes \gamma \in V_{1}$. We know that

$$
F(v \otimes \beta, w \otimes \gamma)=\frac{1}{2} Q(\beta, \gamma)\left\{\left(v w^{*}+w v^{*}\right) \otimes 1+\left(i w v^{*}-i v w^{*}\right) \otimes i\right\},
$$

where $Q$ is a positive Hermitian form on $\mathbf{C}^{p}$.

Thus we find

$$
\begin{aligned}
(r \otimes \alpha \otimes 1+ & i r \otimes \alpha \otimes i) \circ F(v \otimes \beta, w \otimes \gamma)=F(S(r \otimes \alpha)(v \otimes \beta), w \otimes \gamma) \\
= & \frac{1}{2} Q(\beta, \gamma)\left\{w v^{*} r \otimes \alpha \otimes 1+i w v^{*} r \otimes \alpha \otimes i\right\} \\
& =\frac{1}{2} w \otimes \alpha Q \otimes(\beta, \gamma)\left(r^{*} v\right)+\frac{i}{2} w \otimes \alpha \otimes i Q(\beta, \gamma)\left(r^{*} v\right) \\
& =\frac{1}{2} w \otimes \alpha \otimes Q\left(\left(r^{*} v\right) \beta, \gamma\right)+\frac{i}{2} w \otimes \alpha \otimes i Q\left(\left(r^{*} v\right) \beta, \gamma\right) .
\end{aligned}
$$

Notice that the formula above determines $F$ on $V_{0} \times V_{1}$ solely in terms of Jordan algebra $Q$, and the values of $F$ on $V_{1} \times V_{1}$. Moreover, since $F\left(v_{0}, v_{1}\right)=0$ for all $v_{1} \in V_{1}$ if and only if $v_{0}=0$, we see that $\Sigma_{\nu} S\left(r_{\nu} \otimes \alpha_{\nu}\right)\left(v_{\nu} \otimes \beta_{\nu}\right)=0$ if and only if $\Sigma_{\nu} \alpha_{\nu} \otimes Q\left(\beta_{\nu}, \gamma\right) r_{\nu}^{*} v_{\nu}=0$ for all $\gamma \in \mathbf{C}^{p}$. It follows that we may identify $V_{0}$ with $\mathbf{R}^{q} \otimes_{\mathbf{R}} \mathbf{C}^{p}$ in such a fashion that $S(r \otimes \alpha)(v \otimes \beta)=\alpha \otimes\left(r^{*} v\right) \beta$.

Let $(\Theta, \Omega, V, S, F)$ be an incomplete data set with $\mathbb{Q}_{1 / 2}$ and $V_{1}$ identified as above. Then

THEOREM. For any choice of $F$ there exists (uniquely) an admissible completion if and only if $V_{0}$ may be identified with $\mathbf{R}^{q} \otimes_{\mathbf{R}} \mathbf{C}^{p}$ in such a fashion that $S(r \otimes \alpha)(v \otimes \beta)$ $=\alpha \otimes\left(r^{*} v\right) \beta$.

(In case (ii), the identification of $\Theta_{1 / 2}$ and the action of $\Theta_{1}$ on $\Theta_{1 / 2}$ being the same, the corresponding statement is that $V_{0}$ may be identified with $\mathbf{R}^{q} \otimes_{\mathbf{R}} \mathbf{C}^{p}$ in such a fashion that $S(r \otimes \alpha)(v \otimes \beta)=\alpha \otimes\left(r^{t} v\right) \beta$.)

The necessity has already been shown. As for the sufficiency, our hypotheses enable us to well define $F$ on $V_{0} \times V_{1}$ by

$$
\begin{aligned}
F(S(r \otimes \alpha)(v \otimes \beta), w \otimes \gamma)= & \frac{1}{2} w \otimes \alpha \otimes\left(r^{*} v\right) Q(\beta, \gamma) \\
& +\frac{i}{2} w \otimes \alpha \otimes i\left(r^{*} v\right) Q(\beta, \gamma) .
\end{aligned}
$$

$F$ is then defined on $V_{1} \times V_{0}$ by conjugation. Let $s \otimes \delta \in Q_{1 / 2}$. F is defined on $V_{0} \times V_{0}$ by

$$
\begin{aligned}
(s \otimes \delta) & \circ F(S(r \otimes \alpha)(v \otimes \beta), w \otimes \gamma) \\
& =\frac{1}{2} F(S(r \otimes \alpha)(v \otimes \beta), S(s \otimes \delta)(w \otimes \gamma)) \\
& =\frac{1}{2}\left(r^{*} v\right) Q(\beta, \gamma) P(s \otimes \delta, w \otimes \alpha)+\frac{i}{2}\left(r^{*} v\right) Q(\beta, \gamma) P(s \otimes \delta, i w \otimes \alpha) .
\end{aligned}
$$

We have yet to show that $F$ satisfies the appropriate conjugacy relation on $V_{0} \times V_{0}$, that $F$ satisfies the strange identity, and that $F$ is $\Omega$-Hermitian. Now we kncw that for any $x \in Q_{1}$ that $P(x s \otimes \delta, w \otimes \alpha)=P(s \otimes \delta, x w \otimes \alpha)$. Put $f_{1}(s, w)=$ $\left(s^{*} w+w^{*} s\right) / 2$. An argument similar to one we have used before will show that 
there exist uniquely $\theta(\delta, \alpha)$ and $\varphi(\delta, \alpha) \in \mathbb{Q}_{0}$ such that $P(s \otimes \delta, w \otimes \alpha)=$ $f_{1}(s, w) \theta(\delta, \alpha)+f_{1}(s, i w) \varphi(\delta, \alpha)$.

It is now easy to show that the $F$ we have defined satisfies $F\left(v_{0}, v_{0}^{\prime}\right)=\overline{F\left(v_{0}^{\prime}, v_{0}\right)}$.

$F$ restricted to $V_{1} \times V_{1}$ satisfies the strange identity, so we have only to show that $S\left(F\left(v_{1}, v_{0}\right)\right) v_{1}=0$. But it is easy to see that $S(F(w \otimes \gamma), S(r \otimes \alpha)(v \otimes \beta)) \equiv 0$, which is more than enough.

We should also check that for $x \in \mathbb{Q}_{1}$

$$
x \circ F(S(r \otimes \alpha)(v \otimes \beta), w \otimes \gamma)=\frac{1}{2} F(S(r \otimes \alpha)(v \otimes \beta), x w \otimes \gamma),
$$

but this is trivial.

It remains to show that the extended $F$ is $\Omega$-Hermitian. First, take $a=\Sigma_{\nu}\left(r_{\nu} \otimes \alpha_{\nu}\right)$ $\in \mathbb{Q}_{1 / 2}$ and compute $P(a, a)$. We will suppose that $\alpha_{\nu}$ 's are linearly independent in $\mathbf{R}^{q}$. An easy calculation gives $P(a, a)=\Sigma_{\nu, \mu}\left(r_{\nu}^{*} r_{\mu}\right)\left\{\theta\left(\alpha_{\nu}, \alpha_{\mu}\right)+i \varphi\left(\alpha_{\nu}, \alpha_{\mu}\right)\right\}$. Putting for arbitrary scalars $c_{\nu}, r_{\nu}=c_{\nu} r, r \in \mathbf{C}^{n}$, we see that $\theta\left(\alpha_{\nu}, \alpha_{\mu}\right)+i \varphi\left(\alpha_{\nu}, \alpha_{\mu}\right)$ is Hermitian positive in the obvious sense, since $P(a, a)=a^{2} \in \vec{\Omega}_{0}$ and is 0 if and only if $a=0$.

Pick an orthonormal base $\beta_{1}, \beta_{2}, \ldots, \beta_{p}$ for $\mathbf{C}^{p}$ such that $Q\left(\beta_{i}, \beta_{j}\right)=\delta_{i j}$. To show that $F$ is $\Omega$-Hermitian, it will suffice to show that $F(u, u) \in \bar{\Omega}-0$, where $u$ is any nonzero element of $v$ of form $u=v \otimes \beta_{k}+\Sigma_{\nu} S\left(r_{\nu} \otimes \alpha_{\nu}\right)\left(v_{\nu} \otimes \beta_{k}\right)$, or $u=$ $\Sigma_{\nu} S\left(r_{\nu} \otimes \alpha_{\nu}\right)\left(v_{\nu} \otimes \beta_{k}\right)$. Taking the second case first, one calculates that $F(u, u)=\Sigma_{\nu, \mu}\left(r_{\nu}^{*} v_{\nu}\right)\left(v_{\mu}^{*} r_{\mu}\right)\left\{\theta\left(\alpha_{\nu}, \alpha_{\mu}\right)+i \varphi\left(\alpha_{\nu}, \alpha_{\mu}\right)\right\}$. Putting $A_{\nu, \mu}=\left(r_{\nu}^{*} v_{\nu}\right)\left(v_{\mu}^{*} r_{\mu}\right)$, take a spectral decomposition of $A_{\nu, \mu}, A_{\nu, \mu}=\Sigma_{\eta} \lambda_{\eta} c_{\nu}^{\eta} c_{\mu}^{\eta}$, to obtain $F(u, u)=$ $\Sigma_{\eta, \nu, \mu} \lambda_{\eta} c_{\nu}^{\eta} \bar{c}_{\mu}^{\eta}\left\{\theta\left(\alpha_{\nu}, \alpha_{\mu}\right)+i \varphi\left(\alpha_{\nu}, \alpha_{\mu}\right)\right\}$, so $F(u, u) \in \bar{\Omega}_{0} \subset \bar{\Omega}$ and is zero if and only if $\lambda_{\eta}=0 \forall \mu$ which implies $r_{\nu}^{*} v_{\nu}=0 \forall \nu$, which implies $u=0$.

For $u$ of the first form, with $v \neq 0$, another easy calculation gives $\left(v^{*} v\right) F(u, u)=$ $\left[v v^{*}+\sum_{\nu}\left(v v_{\nu}^{*} r_{\nu} \otimes \alpha_{\nu}\right)\right]^{2}$, which belongs to $\bar{\Omega}-0$. The proof of the sufficiency is now completed.

6. We take an admissible data set $(\mathscr{Q}, \Omega, V, S, F)$ with $\mathcal{Q}_{1}$ in case IV. For $V_{1}$ we may take $\mathbf{H}^{2}$, so that for $x \in \mathbb{Q}_{1}, v \in V_{1}, S(x) v=x v$.

We may suppose $\mathbb{Q}_{1 / 2}=\mathbf{H}^{2} \otimes_{\mathbf{R}} \mathbf{R}^{q} \oplus \mathbf{H}^{2} \otimes_{\mathbf{R}} \mathbf{R}^{q^{\prime}}$, and for $x \in \mathbb{Q}_{1}, r \otimes \alpha+s \otimes \delta$ $\in \mathbb{Q}_{1 / 2}, x \circ(r \otimes \alpha+s \otimes \delta)=\frac{1}{2} x r \otimes \alpha+\frac{1}{2} \bar{x} s \otimes \delta$. A lemma of $\S 2$ shows that $S(s \otimes \delta) v=0$. Hence if $w \in V_{1}$, we have $(s \otimes \delta) \circ F(v, w)=0$. As in an earlier argument, the real linear span of $F_{1}(v, w)$, since an ideal in $Q_{1}$, contains $e$. Thus $(s \otimes \delta) \circ e=\frac{1}{2} s \otimes \delta=0$. Hence $q^{\prime}=0$.

The remainder of the discussion of this case is incorporated into the next section.

7. We take an admissible data set with $\mathbb{Q}_{1}$ in case III or IV. For $V_{1}$ we may take $\mathbf{H}^{n}$, and for $x \in \mathbb{Q}_{1}, v \in V_{1}, S(x) v=x v$. For $w \in V_{1}, F(v, w)$ is determined up to real scalar multiple. Accordingly, we may take $F_{1}(v, w)=v w^{*}+w v^{*}$, so that $F(v, w)=F_{1}(v, w) \otimes 1+F_{1}(v, w i) \otimes i$. As invariant Hermitian form $V_{1}$ we take $f(v, w)=w^{*} v-i w^{*} v i$.

Using the result of the last discussion, we may for all $n \geqslant 2$ identify $\mathscr{Q}_{1 / 2}$ with $\mathbf{H}^{n} \otimes_{\mathbf{R}} \mathbf{R}^{q}$ in a manner so that for $x \in \mathbb{Q}_{1}, r \otimes \alpha \in \mathbb{Q}_{1 / 2}, x \circ(r \otimes \alpha)=\frac{1}{2} x r \otimes \alpha$. 
By a lemma of $\S 3$, we know that $S(r \otimes \alpha) v=f(v, r) \lambda(\alpha)+f(v, r j) \rho(\alpha)$ for unique $\lambda(\alpha)$ and $\rho(\alpha)$ in $V_{0}$. Computing $(r \otimes \alpha) \circ F(v, w)=\frac{1}{2} F(S(r \otimes \alpha) v, w)+$ $\frac{1}{2} F(v, S(r \otimes \alpha) w)$ in both ways, and comparing real parts, we obtain

$$
\begin{aligned}
\left(v w^{*} r+w v^{*} r\right) \otimes \alpha= & F_{1}(\lambda(\alpha), w f(r, v))+F_{1}(\rho(\alpha), w f(r j, v)) \\
& +F_{1}(\lambda(\alpha), v f(r, w))+F_{1}(\rho(\alpha), v f(r j, w)) .
\end{aligned}
$$

Put $v=w=r$ to obtain $F_{1}(\lambda(\alpha), v)=\frac{1}{2} v \otimes \alpha$. Put $v=w=r j$ to obtain $F_{1}(\rho(\alpha), v)$ $=-\frac{1}{2} v j \otimes \alpha$. Accordingly, one finds $F(S(r \otimes \alpha) v, w)=\frac{1}{2} w \otimes \alpha \otimes f(v, r)-\frac{1}{2} w i \otimes$ $\alpha \otimes f(v, r i)-\frac{1}{2} w j \otimes \alpha \otimes f(v, r j)-\frac{1}{2} w k \otimes \alpha \otimes f(v, r k)$. Using the same argument as in the last section, one sees that $V_{0}$ may be identified with $\mathbf{R}^{q} \otimes_{\mathbf{R}} \mathbf{H}$, whose $\mathbf{C}$ module structure arises from right action by complex scalars on $\mathbf{H}$, in such a fashion that $S(r \otimes \alpha) v=\alpha \otimes r^{*} v$. This is the end of the story. Indeed:

TheOREM. Let $(\mathbb{Q}, \Omega, V, S, F)$ be an incomplete data set with $\mathbb{Q}_{1}$ in case III or IV. Then with $\Theta_{1 / 2}$ and $V_{1}$ identified as above, the data set nay be completed (uniquely) to an admissible one if and only if $V_{0}$ may be identified with $\mathbf{R}^{q} \otimes_{\mathbf{R}} \mathbf{H}$ in such a fashion that $S(r \otimes \alpha) v=\alpha \otimes r^{*} v$.

We will not give the proof, since it follows very much the same lines as the proof of the last section. The only slightly new ingredients are as follows. Define $g(v, w)=$ $f(v, w)+f(w, v)$. Then one shows by a familiar argument that $P(r \otimes \alpha, s \otimes \beta)$ may be uniquely written $P(r \otimes \alpha, s \otimes \beta)=g(r, s) \theta(\alpha, \beta)+g(r, s i) \varphi(\alpha, \beta)+$ $g(r, s j) \sigma(\alpha, \beta)+g(r, s k) \tau(\alpha, \beta)$. Additionally, one needs the spectral decomposition theorem for $q \times q$ quaternionic Hermitian matrices, which follows readily from the decomposition of the elements of the Jordan algebra of $q \times q$ quaternionic Hermitian matrices into linear combinations of primitive idempotents.

8. The only case remaining is $Q_{1}=\mathbf{R}$, unit element denoted $e$, which is surprisingly the most difficult to analyze. For an admissible data set $(\mathcal{Q}, \Omega, V, S, F)$, we may take $\Theta_{1 / 2}=\mathbf{R}^{q}$, identify $\mathbb{Q}_{1 / 2} \otimes_{\mathbf{R}} \mathbf{C}=\mathbf{C}^{q}$ and for $r \in \mathbb{Q}_{1 / 2}$ take $e \circ r=\frac{1}{2} r$. We may take $V_{1}=\mathbf{C}^{p}$, and for $x \in \mathbb{Q}_{1}, v \in V_{1}, S(x) v=x v$. If $w \in V_{1}$, we may assume $F(v, w)=\langle v, w\rangle e$, where $\langle v, w\rangle$ is a positive Hermitian form on $V_{1}$.

Let $\eta$ be any linear form in the (interior of) dual cone to $\Omega_{0}$, and put $Q(r, s)=$ $\eta(r \circ \bar{s})=\eta(P(r, \bar{s}))$ for $r, s \in \mathbb{Q}_{1 / 2} \otimes C . Q$ is a positive Hermitian form.

For arbitrary $v, w \in V_{1}$ define a linear mapping $L(v, w)$ of $\mathbb{Q}_{1 / 2} \otimes C$ to itself by $L(v, w) r=F(S(r) v, w)$. Then,

Lemma. The adjoint of $L(v, w)$ with respect to $Q$ is $L(w, v)$, and the following identities hold:

$$
\begin{aligned}
& L(v, w) L(v, z)=\langle v, z\rangle L(v, w), \\
& L(z, v) L(w, v)=\langle z, v\rangle L(w, v) .
\end{aligned}
$$

Firstly,

$$
\begin{aligned}
(L(v, w) r) \circ \bar{s} & =\bar{s} \circ F(S(r) v, w)=\frac{1}{2} F(S(r) v, S(s) w) \\
& =r \circ F(v, S(s) w)=r \circ \overline{F(S(s) w, v)}=r \circ \overline{L(w, v) s},
\end{aligned}
$$

which gives adjointness statement. 
Also,

$$
\begin{aligned}
L(v, w) L(v, z) r & =L(v, w) F(S(r) v, z)=F(S(F(S(r) v, z)) v, w) \\
& =F(S(F(S(r) v, z)) v, w)+F(S(F(v, S(\bar{r}) z)) v, w)
\end{aligned}
$$

(because of strange identity) $=F(2 S(r \circ F(v, z)) v, w)=\langle v, z\rangle F(S(r) v, w)=$ $\langle v, z\rangle L(v, w) r$, which is identity (I). (II) follows from (I) by taking adjoints.

The action of the family of operators $L(v, w)$ in the space $Q_{1 / 2} \otimes C=C^{q}$ is obviously fully reducible, since the adjoint of each operator is present in the family. Moreover, the identities (I) and (II) continue to hold when the operators are restricted to any invariant subspace. Accordingly, we will let $l(v, w)$ be a family acting irreducibly on a complex vector space $U$, with the identities (I) and (II) satisfied, and with $l(v, w)$ being linear in the first variable, conjugate linear in the second, for $v$ and $w \in V_{1}=\mathbf{C}^{p}$. Our $L(v, w)$ will then be equivalent to a direct sum, with multiplicities, of the various $l(v, w)$ we find by this process.

Define $U_{\nu}=\Lambda^{\nu} V_{1}, \nu=0,1,2, \ldots, p .\left(U_{0}=\mathbf{C}, U_{1}=V_{1}, U_{2}=V_{1} \wedge V_{1}\right.$, etc. $)$ There is an irreducible representation $\rho_{\nu}$ of the Lie algebra $g l\left(V_{1}\right)$ on $U_{\nu}$ defined by:

$$
\begin{aligned}
\rho_{\nu}(g)\left(v \wedge v^{\prime} \wedge v^{\prime \prime} \wedge \cdots\right)= & g v \wedge v^{\prime} \wedge v^{\prime \prime} \wedge \cdots \\
& +v \wedge g v^{\prime} \wedge v^{\prime \prime} \wedge \cdots+v \wedge v^{\prime} \wedge g v^{\prime \prime} \wedge \cdots+\cdots,
\end{aligned}
$$

where $g \in g l\left(V_{1}\right) . \rho_{0}$ is the trivial representation.

Pick, once and for all, an orthonormal basis, $\alpha_{1}, \alpha_{2}, \ldots, \alpha_{p}$ of $V_{1}$ with respect to $\langle\cdot, \cdot\rangle$. Let $b$ be the conjugation in $V_{1}$ with respect to this choice of basis. Define $\tau(v, w) \in g l\left(V_{1}\right)$ by $\tau(v, w) z=\langle v, b z\rangle b w$. Then it is easy to verify that for any $\nu=0,1,2, \ldots, p, l(v, w)=\rho_{\nu}(\tau(v, w))$ satisfies (I) and (II) and is obviously linear in the first variable, conjugate linear in the second.

THEOREM. The only possibilities for $l(v, w)$ are, up to equivalence (similarity), the $\rho_{\nu}(\tau(v, w)), \nu=0,1,2, \ldots, p$, described above.

For the proof, we take the first identity $l(v, w) l(v, z)=\langle v, z\rangle l(v, w)$, and polarize on $v$ to obtain $l(x, w) l(y, z)+l(y, w) l(x, z)=\langle x, z\rangle l(y, w)+$ $\langle y, z\rangle l(x, w)$. Similarly polarizing the second identity, we get $l(z, x) l(w, y)+$ $l(z, y) l(w, x)=\langle z, x\rangle l(w, y)+\langle z, y\rangle l(w, x)$. In the second polarization, interchange $x$ and $z, y$ and $w$, and subtract from the first to obtain $l(y, w) l(x, z)-$ $l(x, z) l(y, w)=\langle y, z\rangle l(x, w)-\langle x, w\rangle l(y, z)$.

Define $T_{i j}=l\left(\alpha_{j}, \alpha_{i}\right)$, and let $A_{i j}$ be that elementary matrix in $g l\left(\mathbf{C}^{p}\right)$ which has a one in the $(i, j)$ position, zeroes elsewhere. Then our last identity may be rewritten:

$$
T_{i j} T_{k l}-T_{k l} T_{i j}=\delta_{j k} T_{i l}-\delta_{l i} T_{k j} \text {. }
$$

Accordingly, the map $\rho: A_{i j} \rightarrow T_{i j}$ is a Lie algebra representation of $g l\left(\mathbf{C}^{p}\right)$ in End $U$, and by hypothesis, the representation is irreducible. Moreover, the representation restricted to $\mathrm{sl}\left(\mathbf{C}^{p}\right)$ is irreducible; indeed the most general irreducible representation of $g l\left(\mathbf{C}^{p}\right)$ arises by taking one for $\operatorname{sl}\left(\mathbf{C}^{p}\right)$ and extending it to $g l\left(\mathbf{C}^{p}\right)$ by defining the image of $A_{11}+A_{22}+\cdots+A_{p p}$, which must be in the center of the representation, as $\theta \times$ identity, for any fixed choice of complex scalar $\theta$.

We will identify the representation $\rho$ by restricting it to $\operatorname{sl}\left(\mathbf{C}^{p}\right)$ and looking for a weight vector $u$ belonging to a dominant integral weight. In doing so, and also to 
effect the extension to $g l\left(\mathbf{C}^{p}\right)$, it is important to note that $T_{i i}$ is idempotent for all $i$, as follows readily from (I) or (II).

If $\rho$ restricted to $\operatorname{sl}\left(\mathbf{C}^{p}\right)$ is the trivial representation, then

$$
\begin{aligned}
T_{11} & =\rho\left(A_{11}\right) \\
& =\rho\left(A_{11}-\frac{1}{p}\left(A_{11}+A_{22}+A_{p p}\right)\right)+\frac{1}{p} \rho\left(A_{11}+A_{22}+\cdots+A_{p p}\right) \\
& =\theta / p \times \text { identity, }
\end{aligned}
$$

so $T_{11}$ will be idempotent if and only if $\theta / p=0$ or 1 . These two choices correspond to the choices of $\rho_{0}$ on $U_{0}$ or $\rho_{p}$ on $U_{p}$ as described above.

So we may suppose that $\rho$ restricted to $\operatorname{sl}\left(\mathbf{C}^{p}\right)$ is nontrivial. $A_{i}=A_{i i}-A_{i+1, i+1}$, $i=1,2, \ldots, p-1$ form a basis for a Cartan subalgebra, see [3], and we let $u$ be a weight vector for the dominant integral weight of the representation. Put $T_{i}=T_{i i}$ $T_{i+1, i+1}=\rho\left(A_{i}\right)$. Since $u$ is an eigenvector for all $T_{i}$, it is an eigenvector for $T_{1}+2 T_{2}+\cdots+(p-1) T_{p-1}=T_{11}+T_{22}+\cdots+T_{p p}-p T_{p p}$. Since $T_{11}+T_{22}$ $+\cdots+T_{p p}=\theta \times$ identity, $u$ is an eigenvector for $T_{p p}$, hence for all $T_{i i}$. Since $T_{i i}$ is idempotent, $T_{i i} u=\varepsilon_{i} u$ where $\varepsilon_{i}=0$ or 1 . Hence $T_{i} u=\left(\varepsilon_{i}-\varepsilon_{i+1}\right) u=\delta_{i} u$, so $\left(\sum_{1}^{p-1} \lambda_{i} T_{i}\right) u=\sum_{1}^{p-1} \lambda_{i} \delta_{i} u$. $\delta_{i}=0$, 1, or -1 . Since our weight is dominant integral, $\delta_{i}=0$ or 1 . But

$$
\left(T_{1}+T_{2}+\cdots+T_{p-1}\right) u=\left(\delta_{1}+\delta_{2}+\cdots+\delta_{p-1}\right) u=\left(T_{11}-T_{p p}\right) u ;
$$

since $\left(T_{11}-T_{p p}\right) u=0, u$, or $-u$, the only possibilities are that one $\delta_{i}$ is one, the remaining are zeros. Consequently, up to equivalence, $U$ is $U_{1}, U_{2}, \ldots$, or $U_{p-1}$ and $\rho$ is $\rho_{1}, \rho_{2}, \ldots$, or $\rho_{p-1}$, respectively. We have only identified $\rho$ restricted to $\operatorname{sl}\left(\mathbf{C}^{p}\right)$. But it is readily seen that in each case there is one and only one extension to $g l\left(\mathbf{C}^{p}\right)$ such that $T_{11}$ is idempotent, and it is the one described by the theorem, completing the proof.

Consequently, $L(v, w)$ is equivalent to a direct sum of the $\rho_{\nu}(\tau(v, w))$. Put another way, we will identify $\mathcal{U}_{1 / 2} \otimes_{\mathbf{R}} \mathbf{C}$ with $\oplus_{\nu} U_{\nu} \otimes \mathbf{R}^{n_{\nu}}$ in such a way that for $x=u \otimes \alpha$ $\in U_{\nu} \otimes \mathbf{R}^{n_{\nu}}=: W_{\nu}, L(v, w) x=\rho_{\nu}(\tau(v, w)) u \otimes \alpha$.

$L(v, w)$ depends only on $\tau(v, w)$. We will write $L(v, w)=L(\tau(v, w))$, and the new definition of $L$ may be linearly extended unambiguously to all of $g l\left(V_{1}\right)$ by defining $L(g) x=\rho_{\nu}(g) u \otimes \alpha$.

For any $g \in g l\left(V_{1}\right)$ we define its adjoint $g^{*}$ with respect to $\langle\cdot, \cdot\rangle$. It is easy to see that $\tau^{*}(v, w)=\tau(w, v)$, and that $\operatorname{tr} \tau(v, w)=\langle v, w\rangle$.

Let $h$ be the conjugation in $Q_{1 / 2} \otimes_{\mathbf{R}} \mathbf{C}$.

LEMMA. $\operatorname{tr} g-L(g)=h L\left(g^{*}\right) h$.

We know $r \circ F(v, w)=\frac{1}{2}\langle v, w\rangle r=\frac{1}{2} F(S(r) v, w)+F(v, S(h r) w)$ for $r \in \Theta_{1 / 2}$ $\otimes \mathbf{C}$, or

$$
\begin{aligned}
\langle v, w\rangle r & =L(v, w) r+h L(w, v) h r \\
& =L(\tau(v, w)) r+h L(\tau(w, v)) h r
\end{aligned}
$$

since $\operatorname{tr} \tau(v, w)=\langle v, w\rangle$, and since every $g \in g l\left(V_{1}\right)$ may be written as a sum of $\tau(v, w)$ for appropriate choices of $v$ and $w$, the statement of the lemma follows. 
LEMMA. $h$ is a semilinear bijection of $W_{\nu}$ to $W_{p-\nu}, \nu=0,1, \ldots, p$.

For $w \in W_{\nu}, \rho_{\nu}(1) w=\nu w$. The statement now follows trivially from the last lemma.

COROLLARY. $n_{\nu}=n_{p-\nu}$.

(In case $p$ is even, then $h$ is a conjugation of $W_{p / 2}$ satisfying $\operatorname{tr} g-L(g)=$ $h L\left(g^{*}\right) h$. Such conjugations do not always exist, for example if $p=2, n_{1}=1$; we do not explore the phenomenon.)

Lemma. $P(L(g) r, s)=P\left(r, h L\left(g^{*}\right) h s\right)=(\operatorname{tr} g) P(r, s)-P(r, L(g) s)$. Put $\tilde{P}(r, s)=P(r, h s)$. Then $\tilde{P}(L(g) r, s)=\tilde{P}\left(r, L\left(g^{*}\right) s\right)$.

We know

$$
\begin{aligned}
P(L(\tau(v, w)) r, s) & =P(L(v, w) r, s)=s \circ F(S(r) v, w)=\frac{1}{2} F(S(r) v, S(h s) w) \\
& =r \circ h F(S(h s) w, v)=r \circ h L(\tau(w, v) h s) .
\end{aligned}
$$

Since $\tau^{*}(v, w)=\tau(w, v)$, the first statement follows. The remaining statements are now obvious.

Form a positive Hermitian form $Q$ on $\mathbb{Q}_{1 / 2} \otimes \mathbf{C}$ as before; $Q(\cdot, \cdot)=\eta \tilde{P}(\cdot, \cdot)$. With respect to $Q$, form the adjoint $L^{*}(g)$ of $L(g)$. By virtue of the last lemma we see that $L^{*}$ is independent of $\eta$. Indeed,

Corollary. $L^{*}(g)=L\left(g^{*}\right)$.

Corollary. If $r \in W_{\nu}, s \in W_{\mu}$, then $\tilde{P}(r, s)=0$ unless $\nu=\mu$. Equivalently, $P(r, s)=0$ unless $\nu+\mu=p$.

For the statement $Q(L(g) v, w)=Q\left(v, L\left(g^{*}\right) w\right)$, when restricted to $W_{\nu} \times W_{\mu}$ says the two representations $\rho_{\nu}$ and $\rho_{\mu}$ are equivalent, which holds only for $\nu=\mu$.

We are now almost ready to identify $V_{0}$, but we need a few more facts. Let $r \in U_{\nu}$. $r$ may be regarded as a skew multilinear form on $\nu$ vectors drawn from the dual space of $V_{1}$, which we may identify with $V_{1}$ using the symmetric nondegenerate bilinear form $(v, w)=\langle v, b w\rangle$. With these conventions,

$$
\begin{aligned}
\left(\rho_{\nu}(g) r\right)\left(u_{1}, u_{2}, \ldots, u_{\nu}\right)= & r\left(b g^{*} b u_{1}, u_{2}, \ldots, u_{\nu}\right)+r\left(u_{1}, b g^{*} b u_{2}, \ldots, u_{\nu}\right)+\cdots \\
& +r\left(u_{1}, u_{2}, \ldots, b g^{*} b u_{\nu}\right) .
\end{aligned}
$$

In particular,

$$
\begin{aligned}
\left(\rho_{\nu}(\tau(v, w)) r\right)\left(u_{1}, u_{2}, \ldots, u_{\nu}\right)= & \left\langle u_{1}, w\right\rangle r\left(v, u_{2}, \ldots, u_{\nu}\right)+\left\langle u_{2}, w\right\rangle r\left(u_{1}, v, \ldots, u_{\nu}\right) \\
& +\cdots+\left\langle u_{\nu}, w\right\rangle r\left(u_{1}, u_{2}, \ldots, v\right) .
\end{aligned}
$$

For any $r \in U_{\nu}$, and $v \in V_{1}$, we define $r[v] \in U_{\nu-1}$ by $r[v]\left(u_{2}, u_{3}, \ldots, u_{\nu}\right)=$ $r\left(v, u_{2}, u_{3}, \ldots, u_{v}\right)$. (If $r \in U_{0}, r[v]=0$.) The definition of $r[v]$ is extended by linearity to any $s \in \mathbb{Q}_{1 / 2} \otimes_{\mathbf{R}} \mathbf{C}$ (thus, $\left.(r \otimes \alpha)[v]=r[v] \otimes \alpha\right)$ to give a map of $\left(Q_{1 / 2} \otimes C\right) \times V_{1}$ into $\oplus_{1}^{p} U_{\nu-1} \otimes \mathbf{R}^{n_{\nu}}$.

Lemma. Let $s_{j} \in \mathbb{Q}_{1 / 2} \otimes \mathbf{C}, v_{j} \in V_{1}$. Then $\Sigma_{j} S\left(s_{j}\right) v_{j}=0$ if and only if $\Sigma s_{j}\left[v_{j}\right]=0$. 
$\Sigma_{j} S\left(s_{j}\right) v_{j}=0$ if and only if $\Sigma_{j} F\left(S\left(s_{j}\right) v_{j}, w\right)=0$ for all $w \in V_{1}$, i.e. if and only if $\Sigma_{j} L\left(v_{j}, w\right) s_{j}=0$ for all $w$. For each $\nu$, let $\alpha_{\nu}^{i}, i=1,2, \ldots, n_{\nu}$ be an independent basis for $\mathbf{R}^{n_{\nu}}$, and write $s_{j}=\Sigma_{\nu, i}\left(r_{\nu}^{i j} \otimes \alpha_{\nu}^{i}\right)$, where $r_{\nu}^{i j} \in U_{\nu}$. Then $\Sigma_{j} L\left(v_{j}, w\right) s_{j}=$ $\Sigma_{\nu, i, j}\left(\rho_{\nu}\left(\tau\left(v_{j}, w\right)\right) r_{\nu}^{i j} \otimes \alpha_{\nu}^{i}\right)=0$ if and only if $\Sigma_{j} \rho_{\nu}\left(\tau\left(v_{j}, w\right)\right) r_{\nu}^{i j}=0$ for all $\nu, i$, and $w$.

Let $r \in U_{\nu}$. Then

$$
\begin{aligned}
\left(\rho_{\nu}(\tau(v, w)) r\right)\left(u_{1}, u_{2}, \ldots, u_{\nu}\right)= & \left\langle u_{1}, w\right\rangle r\left(v, u_{2}, \ldots, u_{\nu}\right)+\cdots \\
& +\left\langle u_{\nu}, w\right\rangle r\left(u_{1}, u_{2}, \ldots, u_{\nu-1}, v\right) .
\end{aligned}
$$

Thus

$$
\begin{aligned}
\left(\sum_{j} \rho_{\nu}\left(\tau\left(v_{j}, w\right) r_{\nu}^{i j}\right)\right)\left(u_{1}, u_{2}, \ldots, u_{\nu}\right)= & \left\langle u_{1}, w\right\rangle \sum_{j} r_{\nu}^{i j}\left(v_{j}, u_{2}, \ldots, u_{\nu}\right) \\
& +\cdots+\left\langle u_{\nu}, w\right\rangle \sum_{j} r_{\nu}^{i j}\left(u_{1}, u_{2}, \ldots, u_{\nu-1}, v_{j}\right) .
\end{aligned}
$$

Pick $w$ a nonzero vector orthogonal to $u_{2}, u_{3}, \ldots, u_{v}$ and put $u_{1}=w$ to see that $\Sigma_{j} S\left(s_{j}\right) v_{j}=0$ implies $\Sigma_{j} s_{j}\left[v_{j}\right]=0$. The implication in the other direction is straightforward.

The last lemma clearly tells us what $V_{0}$ must be.

THEOREM. $V_{0}$ may be identified with $\oplus_{1}^{p} U_{\nu-1} \otimes \mathbf{R}^{n_{\nu}}$ in such a fashion that for $s \in \mathbb{Q}_{1 / 2} \otimes \mathbf{C}, v \in V_{1}, S(s) v=s[v]$.

It is only necessary to remark that our definition of $V_{0}$ is not too large. But if $r \in U_{\nu-1}$, it is easy to see that there exist $r_{j} \in U_{\nu}$ such that $\sum_{1}^{p} r_{j}\left[\alpha_{j}\right]=r$.

THEOREM. Let $(\Theta, \Omega, V, S, F)$ be an incomplete data set with $\mathscr{Q}_{1}=\mathbf{R}$. Let $U_{v}=\Lambda^{\nu} V_{1}$, and let $h$ be the conjugation in $\mathbb{Q}_{1 / 2} \otimes_{\mathbf{R}} \mathbf{C}$. Let $\tilde{P}(r, s)=P(r, h s)$, for $r, s \in \mathbb{Q}_{1 / 2}$ $\otimes_{\mathbf{R}} \mathbf{C}$. The data set may be completed to an admissible one if and only if:

(1) $\mathbb{Q}_{1 / 2} \otimes \mathbf{C}$ may be identified with $\oplus_{0}^{p} U_{\nu} \otimes \mathbf{R}^{n_{\nu}}$, and $V_{0}$ may be identified with $\bigoplus_{1}^{p} U_{\nu-1} \otimes \mathbf{R}^{n_{\nu}}$ in such a fashion that $S(s) v=s[v]$ for $s \in \mathbb{Q}_{1 / 2} \otimes \mathbf{C}, v \in V_{1}$,

(2) if $L(g)$ has for all $g \in g l\left(V_{1}\right)$ the usual action on $\mathbb{Q}_{1 / 2} \otimes \mathbf{C}$, then $\operatorname{tr} g-L(g)=$ $h L\left(g^{*}\right) h$, where $g^{*}$ is the adjoint of $g$ with respect to the Hermitian form $F(\cdot, \cdot)$ on $V_{1}$; and

(3) $\tilde{P}(L(g) r, s)=\tilde{P}\left(r, L\left(g^{*}\right) s\right)$ for $r, s \in \mathbb{Q}_{1 / 2} \otimes \mathbf{C}$.

The necessity has already been proved.

Before the proof of sufficiency, a word or two about the hypotheses is in order. The reader must remember that the definition of $r[v]$ depended on an identification of the dual space of $V_{1}$ with $V_{1}$ itself via the conjugation $b$ arising from a choice $\alpha_{1}, \alpha_{2}, \ldots, \alpha_{p}$ of orthonormal basis in $V_{1}$. Bearing this in mind, we define for $v, w \in V_{1}, \tau(v, w) \in g l\left(V_{1}\right)$ by $\tau(v, w) z=\langle v, b z\rangle b w$.

Next we define for $r \in \mathbb{Q}_{1 / 2} \otimes \mathbf{C}, v, w \in V_{1}, F(S(r) v, w)=L(\tau(v, w)) r$, which extends unambiguously to a definition of $F$ on $V_{0} \times V_{1}$ by virtue of hypothesis (1).

$F$ is now defined on $V_{1} \times V_{0}$ using the conjugation $h$. The hypothesis (2) now leads immediately to the conclusion that $r \circ F(v, w)=\frac{1}{2} F(S(r) v, w)$ $+\frac{1}{2} F(v, S(h r) w)$. 
The last conclusion, coupled with the observations that (i) $L(\tau(v, w)) L(\tau(v, z))$ $=\langle v, z\rangle L(\tau(v, w))$ and (ii) $L(\tau(v, w)) r=0$ for all $w$ implies $S(r) v=0$, gives us the statement $S(F(v, S(r) w)) v \equiv 0$, so $F$ will satisfy the strange identity.

- $F$ is now defined on $V_{0} \times V_{0}$ by setting $F(S(r) v, S(s) w)=2(h s) \circ F(S(r) v, w)$ $=2 P(h s, L(\tau(v, w)) r)$. We must verify that $F$ satisfies the appropriate conjugacy relation on $V_{0} \times V_{0}$. It suffices to show that $F(S(r) v, S(s) w)=\overline{F(S(s) w, S(r) v)}$, where the conjugation is in $\mathbb{Q}_{0} \otimes_{\mathbf{R}} \mathbf{C}$. This requires $P(h s, L(\tau(v, w)) r)=$ $\bar{P}(h r, L(\tau(w, v)) s)=P(r, h L(\tau(w, v)) s)$, which is immediate by (3).

It remains to show that the $F$ we have defined is $\Omega$-Hermitian. A few observations are needed first. From the polarized form of (I) we obtain readily

$$
\langle w, w\rangle L(x, y)-L(w, y) L(x, w)=L(x, y) L(w, w)-\langle x, w\rangle L(w, y)
$$

where we are defining $L(v, w)$ as $L(\tau(v, w))$. Put $w=\alpha_{k}$, and sum on $k$ to obtain

$$
\sum_{1}^{p} L\left(\alpha_{k}, y\right) L\left(x, \alpha_{k}\right)=(p+1) L(x, y)-L(x, y) L(1) .
$$

Now we select $u \in V_{0}$ of form $u=\Sigma_{i} S\left(r_{i}\right) v_{i}$, and we must show $F(u, u) \in \bar{\Omega}$ and is zero if and only if $u=0$. But $F(u, u)=\sum_{i, j} F\left(S\left(r_{i}\right) v_{i}, S\left(r_{j}\right) v_{j}\right)=$ $2 \Sigma_{i, j} h r_{j} \circ F\left(S\left(r_{i}\right) v_{i}, v_{j}\right)=2 \sum_{i, j} \tilde{P}\left(L\left(v_{i}, v_{j}\right) r_{i}, r_{j}\right)$.

The hypothesis (3) of our theorem clearly implies that $\tilde{P}(r, s)$, for $r \in W_{\nu}, s \in W_{\mu}$ is zero unless $\nu=\mu$. So in order to establish our claim, it suffices to assume that $r_{i} \in W_{\nu}$ for all $i$. But then

$$
\begin{aligned}
\sum_{i, j} \tilde{P}\left(L\left(v_{i}, v_{j}\right) r_{i}, r_{j}\right) & =\frac{1}{p+1-\nu} \sum_{i, j, k} \tilde{P}\left(L\left(\alpha_{k}, v_{j}\right) L\left(v_{i}, \alpha_{k}\right) r_{i}, r_{j}\right) \\
& =\frac{1}{p+1-\nu} \sum_{i, j, k} \tilde{P}\left(L\left(v_{i}, \alpha_{k}\right) r_{i}, L\left(v_{j}, \alpha_{k}\right) r_{j}\right) \\
& =\frac{1}{p+1-\nu} \sum_{k} \tilde{P}\left(s_{k}, s_{k}\right) \quad \text { where } s_{k}=\sum_{i} L\left(v_{i}, \alpha_{k}\right) r_{i} .
\end{aligned}
$$

Hence $F(u, u) \in \bar{\Omega}$ and is zero if and only if $s_{k}=0 \forall k$. But the last is equivalent to $\Sigma_{i} L\left(v_{i}, w\right) r_{i}=0 \forall w$; which is equivalent to $\Sigma_{i} r_{i}\left[v_{i}\right]=0$, i.e. $\Sigma_{i} S\left(r_{i}\right) v_{i}=0$.

Finally, we take $u \in V_{1} \oplus V_{0}$ of form $u=w+\Sigma_{i} S\left(r_{i}\right) v_{i}$, with $w \neq 0$, and must show $F(u, u) \in \bar{\Omega}$. Now $F(u, u)=\langle w, w\rangle e+\sum_{i} L\left(v_{i}, w\right) r_{i}+\sum_{i} h L\left(v_{j}, w\right) r_{i}+$ $2 \Sigma_{i, j} \tilde{P}\left(L\left(v_{i}, v_{j}\right) r_{i}, r_{j}\right)=x+r+k, x=\langle w, w\rangle e \in \Omega_{1}, r \in Q_{1 / 2}, k \in \mathbb{Q}_{0}$, which $\in \bar{\Omega}$ if and only if $k \geqslant P(r, r) /\langle w, w\rangle$.

For the case at hand, this reduces to showing that

$$
\begin{aligned}
\langle w, w\rangle \sum_{i, j} \tilde{P}\left(L\left(v_{i}, v_{j}\right) r_{i}, r_{j}\right) & \geqslant \sum_{i, j} \tilde{P}\left(L\left(v_{i}, w\right) r_{i}, L\left(v_{j}, w\right) r_{j}\right) \\
& =\sum_{i, j} \tilde{P}\left(L\left(w, v_{j}\right) L\left(v_{i}, w\right) r_{i}, r_{j}\right) .
\end{aligned}
$$

Using $\left(\mathrm{I}^{\prime}\right)$, we have the equivalent inequality:

$$
\sum_{i, j} \tilde{P}\left(L\left(v_{i}, v_{j}\right) L(w, w) r_{i}, r_{j}\right) \geqslant \sum_{i, j}\left\langle v_{i}, w\right\rangle \tilde{P}\left(L\left(w, v_{j}\right) r_{i}, r_{j}\right)
$$


In the last, replace each $v_{i}$ by a multiple of $w$ plus a vector $v_{i}^{\prime}$ orthogonal to $w$ to obtain

$$
\sum_{i, j} \tilde{P}\left(L\left(v_{i}^{\prime}, v_{j}^{\prime}\right) L(w, w) r_{i}, r_{j}\right) \geqslant 0 .
$$

But the construction of the $v_{i}^{\prime}$ implies that $L\left(v_{i}^{\prime}, v_{j}^{\prime}\right)$ commutes with $L(w, w)$. Hence:

$$
\sum_{i, j} \tilde{P}\left(L\left(v_{i}^{\prime}, v_{j}^{\prime}\right) L(w, w) r_{i}, r_{j}\right)=\frac{1}{\langle w, w\rangle} \sum_{i, j} \tilde{P}\left(L\left(v_{i}^{\prime}, v_{j}^{\prime}\right) L(w, w) r_{i}, L(w, w) r_{j}\right)
$$

and assuming, as we may without losing generality, that all $r_{i} \in W_{\nu}$, the last is equal to

$$
\frac{1}{\langle w, w\rangle(p+1-\nu)} \sum_{i, j, k} \tilde{P}\left(L\left(\alpha_{k}, v_{j}^{\prime}\right) L\left(v_{i}^{\prime}, \alpha_{k}\right) L(w, w) r_{i}, L(w, w) r_{j}\right)
$$

which is obviously nonnegative. This completes the proof of the theorem.

It is probably worth noting that the proof that $F$ is $\Omega$-Hermitian can also be reduced to a more concrete and conventional computation by the following device. There is only one (up to complex multiple) sesquilinear form $(\cdot, \cdot)$ on $U_{\nu} \times U_{\nu}$ which satisfies $(L(g) r, s)=\left(r, L\left(g^{*}\right) s\right)$; in fact just the extension to $U_{\nu}$ of the scalar product $\langle\cdot, \cdot\rangle$ in $V_{1}$. Once this observation is made $\tilde{P}$ can be determined more or less explicitly, and the proof reduces to standard, but long, calculations in multilinear algebra.

Finally

THEOREM. The completion of the last theorem is unique.

We have to show that there is only one extension of $F$. This will follow from the following.

Lemma. For each $\nu$, let $\alpha_{\nu}^{i}, i=1,2, \ldots, n_{\nu}$, be independent basis for $\mathbf{R}^{n{ }}$. Let $s=\Sigma_{t, v} r_{\nu}^{i} \otimes \alpha_{v}^{i} \in \mathbb{U}_{1 / 2}^{\prime} \otimes \mathbf{C}$ and let $v \neq 0 \in V_{1}$. Then $S(h s) v=0$ if and only if each $r_{v}^{i}$ (considered as skew multilinear form) vanishes when all of its arguments are orthogonal to $v$.

$$
\begin{aligned}
S(h s) v & =0 \Leftrightarrow F(S(h s) v, w)=0 \forall w \in V_{1} \\
& \Leftrightarrow L(v, w) h s=0 \forall w \in V_{1} \Leftrightarrow \rho_{\nu}(\tau(w, v)) r_{\nu}^{i}=\langle w, v\rangle r_{\nu}^{i}
\end{aligned}
$$

for all $i, \nu$ and $w \in V_{1}$, the last implication using the fact that $\operatorname{tr} g-L(g)=h L\left(g^{*}\right) h$. Fix $i$ and $\nu$ for the moment and call $r_{\nu}^{i}=r$. Then

$$
\begin{aligned}
& \left(\rho_{\boldsymbol{d}^{\prime}}(\tau(w, v)) r\right)\left(u_{1}, u_{2}, \ldots, u_{\nu}\right)=\left\langle u_{1}, v\right\rangle r\left(w, u_{2}, \ldots, u_{\nu}\right) \\
& +\cdots+\left\langle u_{\nu}, v\right\rangle r\left(u_{1}, u_{2}, \ldots, u_{\nu-1}, w\right)=\langle w, v\rangle r .
\end{aligned}
$$

Pick $u_{2}, u_{3}, \ldots, u_{v}$ orthogonal to $v, u_{1}=v$, and $w$ orthogonal to $v$ to obtain $r\left(w, u_{2}, \ldots, u_{v}\right)=0$. So $r$ vanishes when its arguments are orthogonal to $v$. But if $r$ vanishes when its arguments are orthogonal to $v$, write each $u_{i}$ and $w$ as a multiple of $v$ plus a vector orthogonal to $v$ to see that

$$
\left(\rho_{\nu}(\tau(w, v)) r\right)\left(u_{1}, u_{2}, \ldots, u_{\nu}\right)=\langle w, v\rangle r\left(u_{1}, u_{2}, \ldots, u_{\nu}\right),
$$

completing the proof. 
Lemma. Given any $r \in \mathbb{Q}_{1 / 2} \otimes \mathbf{C}$ and any $v \neq 0$ in $V_{1}$, there is a unique $s \in \mathbb{Q}_{1 / 2} \otimes$ C such that $S(s) v=0$ and $S(h s) v=S(h r) v$.

Write $r=\Sigma_{i, \nu} r_{\nu}^{i} \otimes \alpha_{\nu}^{i}$ and $s=\sum_{i, \nu} s_{\nu}^{i} \otimes \alpha_{\nu}^{i}$. But then $S(s) v=0$ tells us, as we know from a previous lemma, that each $s_{v}^{i}$ vanishes when its first argument is set equal to $v$, while $S(h s-h r) v=0$ says each $s_{\nu}^{i}$ takes the same values as $r_{\nu}^{i}$ when all the arguments are orthogonal to $v$. These requirements fix $s_{v}^{i}$ uniquely and complete the proof of the lemma.

To see the proof of the theorem, suppose $r \in \mathbb{Q}_{1 / 2} \otimes \mathbf{C}$ and $v \neq 0$ in $V_{1}$ are given. Find the $s$ of the last lemma. Then $F(S(r) v, v)=2(r-s) \circ F(v, v)=\langle v, v\rangle(r-s)$ is uniquely determined. Similarly $F(S(r) w, w), F(S(r)(v+i w),(v+i w))$ and $F(S(r)(v-i w))$ are uniquely determined. But an appropriate linear combination of these four gives $F(S(r) v, w)$.

My thanks go to the referee for correcting several mistakes in the first draft of this manuscript.

\section{REFERENCES}

1. J. Dorfmeister, Homogene Siegel-Gebeite, Habilitationschrift, Westfälischen Wilhelms Universität zu Münster, 1979.

2. F. and N. Jacobson, Classification and representation of semi-simple Jordan algebras, Trans. Amer. Math. Soc. 65 (1949), 141-169.

3. N. Jacobson, Lie algebras, Interscience, New York, 1962.

4. W. Kaup, Einige bemerkungen über polynomiale Vectorfelder, Jordan algebren, und die Automorphismen von Siegelschen Gebieten, Math. Ann. 204 (1973), 131-144.

5. W. Kaup, Y. Matsushima and T. Ochiai, On the automorphisms and equivalences of generalized Siegel domains, Amer. J. Math. 92 (1970), 475-479.

6. O. Ruthaus, Automorphisms of Siegel domains, Trans. Amer. Math. Soc. 162 (1971), 351-382.

7. __ Ordered Jordan algebras, Amer. J. Math. 100 (1978), 925-939.

8. __ Automorphisms of Siegel domains, Amer. J. Math. 101 (1979), 1167-1179.

9. I. Satake, Algebraic structure of symmetric domains, Princeton Univ. Press, Princeton, N. J., 1980.

Department of Mathematics, Cornell University, Ithaca, New York 14853 